ISSN: 2052-5206

journals.iucr.org/b

\title{
Is there any point in making co-crystals?
}

\section{Christer Aakeröy}

Acta Cryst. (2015). B71, 387-391

\section{IU IUCr Journals CRYSTALLOGRAPHY JOURNALS ONLINE}

Copyright (C) International Union of Crystallography

Author(s) of this paper may load this reprint on their own web site or institutional repository provided that this cover page is retained. Republication of this article or its storage in electronic databases other than as specified above is not permitted without prior permission in writing from the IUCr.

For further information see http://journals.iucr.org/services/authorrights.html 
STRUCTURAL SCIENCE CRYSTAL ENGINEERING MATERIALS

ISSN 2052-5206

Received 6 May 2015

Accepted 5 June 2015

Edited by A. J. Blake, University of Nottingham, UK

Keywords: co-crystals; crystal engineering; hydrogen bonds; halogen bonds.

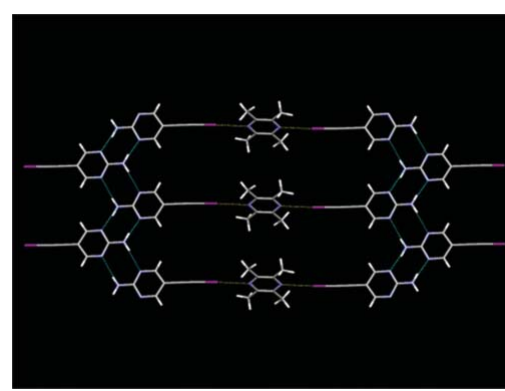

C 2015 International Union of Crystallography

\section{Is there any point in making co-crystals?}

\author{
Christer Aakeröy*
}

Department of Chemistry, Kansas State University, CBC 213, Manhattan, Kansas 66506, United States. *Correspondence e-mail: aakeroy@ksu.edu

Many aspects of co-crystals, including their synthesis, characterization and possible applications, are receiving considerable attention from academia and industry alike. The question is, can this interdisciplinary activity be translated into new fundamental insight and new solid forms of high-value materials with improved performances.

\section{Introduction}

Given the extensive debate on how to deal with the term 'cocrystal', be it from a chemical or a legal perspective (Stahly, 2009; Childs \& Zaworotko, 2009; Desiraju, 2003; Dunitz, 2003), it is probably an act of folly to include the term in the title of any manuscript, let alone in one that aims to briefly address the possible impact that co-crystals and co-crystallizations may have on fundamental and applied chemistry.

In a Highlight article published some ten years ago (Aakeröy \& Salmon, 2005), the authors did provide the following statement as part of the introduction: 'The purpose of this article is not to propose new definitions ... but it will be necessary to delineate the scientific realm of this Highlight.' As it happened, the attempt to 'delineate' the focus of the article must have been executed rather poorly as it was subsequently taken out of context on several occasions and interpreted as an effort to propose an actual definition of the term 'co-crystal'. Although some readers did not have any problems making the distinction, as Bond (2007) pointed out, "... the authors were careful to note that their listed characteristics were not 'new definitions'", it may now be wise to resist any temptation to proffer new definitions or article outlines lest another unintentional contribution is made to the general discussion of the semantics of the term 'co-crystal'. Maybe it is pertinent to recall the words of United States Supreme Court Justice Potter Stewart who in 1964 declared (albeit on a very different matter) that 'I shall not today attempt further to define the kinds of material I understand to be embraced within that shorthand description... But I know it when I see it,...' (Jacobellis versus Ohio, 1964). It is probably fair to say that most experienced practitioners of crystal engineering and co-crystal synthesis actually have a pretty good idea of what a co-crystal is even though they are occasionally faced with a structure or an example that presents a real conundrum and a challenge to those ideas.

Leaving all aspects of co-crystal etymology and glossology behind, it is beyond dispute that the number of publications (as well as citations thereof) involving either fundamental or applied aspects of co-crystals has grown exponentially in the last two decades, Fig. 1. 


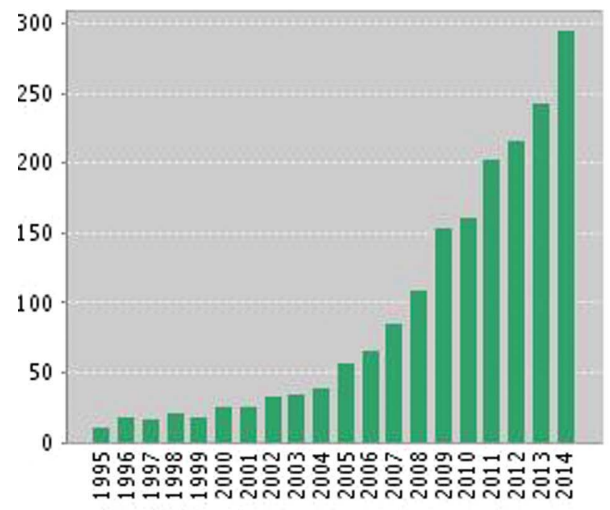

(a)

Figure 1

(a) Published articles on co-crystals 1995-2014. (b) Citations for articles on co-crystals 1995-2015 (Source: Web of Science ${ }^{\circledR}$, April 2015).

This then brings us back to the title of this paper; even though a rapidly increasing number of studies that are more or less connected to co-crystals are being presented each year, is there any point in making co-crystals? Has this flurry of activity offered new information that has improved or enhanced our understanding of intermolecular forces, molecular recognition events, nucleation, crystallization and practical crystal engineering? Are these fundamental efforts creating new scientific opportunities that can positively impact and be of interest to any of the applied sciences communities?

\section{Context}

The history of co-crystals can be traced back to Wöhler's work in the mid-19th century on quinone and hydroquinone, but as a focused and readily identifiable research area it owes a lot to Etter's groundbreaking work on co-crystals some 25 years ago (Etter, 1990) and Desiraju's seminal book on crystal engineering (Desiraju, 1989).

These contributions undoubtedly inspired many organic solid-state chemists, and even if the increase in the number of articles in this field were calibrated against the simultaneous expansion of chemistry journal pages that are published each year, there is no doubt that interest in the synthesis, characterization and application of co-crystals has grown extremely rapidly, and there are no signs yet to indicate that the field has reached a plateau or that it has become saturated. One of the basic driving forces behind research in this area is the recognition that the crystal structure of a compound, the three-dimensional orientation and organization of molecules in a highly regular manner, ultimately determines many fundamental physical properties of that particular material, e.g. thermal stability, hygroscopicity, density and mechanical strength. Therefore, if a purposeful change of the metrics and topologies of the crystalline environment can be achieved by taking full advantage of the directionality and selectivity inherent in many non-covalent interactions, then the design and preparation of materials with tailored physical properties may be achieved within the context of co-crystal synthesis.

So what kind of fundamental new insight and understanding has this burst of activity generated? First, it is worth remembering that the deceptively simple act of molecular recognition, which produces the essential starting point and subsequent driving force for any successful supramolecular assembly, is a result of a delicate balancing act between relatively weak, reversible and highly complex interactions. It is therefore a testament to the progress of crystal engineering in general and co-crystal synthesis in particular that we are today often in a position to confidently say, when looking at a couple of molecules that, 'Yes, I am quite sure that those particular molecules will come together to form a cocrystal that contains supramolecular dimers/trimers/infinite chains/ribbons/layers', Fig. 2.

On a fundamental level, it is clear that concepts such as 'tectons' (Wuest, 2001) and 'synthons' (Desiraju, 1995), both of which represent a potent distillate from extensive crystallographic data, have been fully embraced by the co-crystal community and incorporated into effective synthetic strategies for the assembly of heteromeric organic solids. As a result of this relatively new yet robust and versatile expertise that has
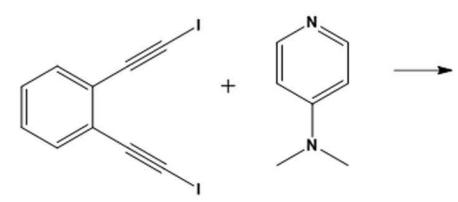

(a)

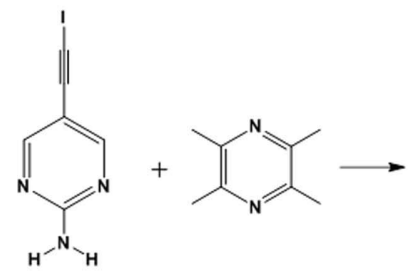

(b)
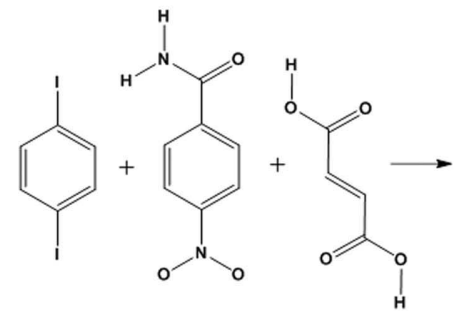

(c)

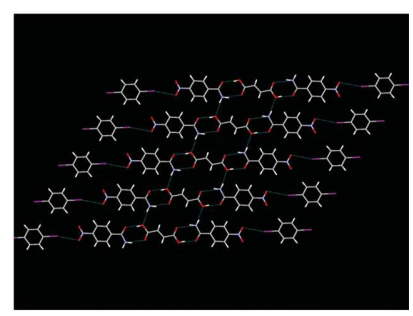

Figure 2

Examples of motifs in co-crystals assembled using clear synthetic protocols: (a) a halogen-bonded trimer (Bosch, 2014); (b) a hydrogenand halogen-bonded layer (Aakeröy, Wijethunga et al., 2015); (c) a ternary hydrogen- and halogen-bonded layer (Tothadi \& Desiraju, 2013). 
been established, it also seems that the co-crystal community is occasionally a victim of its own success. Many manuscripts and research proposals from this field are undeservedly labelled as being 'routine' just because the results or the planned work look quite simple and logical. However, these things only look 'simple' now with the benefit of hindsight because the community has delivered transferable know-how and understanding in an unusually short time frame. It may be that casual remarks along the lines of 'well, that's a pretty obvious result!' offer the greatest indication that our understanding of solid-state assembly and the practical ramifications of directional intermolecular forces have all been greatly advanced as a direct result of the experimental and theoretical studies on co-crystals that have been presented in the last 25 years.

The examples presented in Fig. 2 display motifs that may seem straightforward or even self-evident but it is only relatively recently that we have been able to identify reliable trends and pattern-preferences among different functional groups through access to a sufficient number of organic smallmolecule crystal structures. The Cambridge Structural Database (CSD; Allen, 2002), which is maintained and continuously developed by the Cambridge Crystallographic Data Centre (CCDC), remains an essential research tool in this area, and it is interesting to note how the increase in the number of deposited crystal structures to the CSD mirrors the rise in publications related to co-crystals, Fig. 3. Furthermore, recent changes to the way in which deposited data is processed have dramatically improved the rate at which new crystallographic data is curated and made available to the CSD, and have ensured that the CCDC can keep pace with the rapidly increasing output from the crystallographic community (Bruno \& Groom, 2014).

\section{Covalent and non-covalent synthesis}

Making co-crystals is clearly an aspect of chemical synthesis and it is therefore inevitable that comparisons between covalent and non-covalent synthesis will be made. Organic transformations are achieved with the help of a large library of

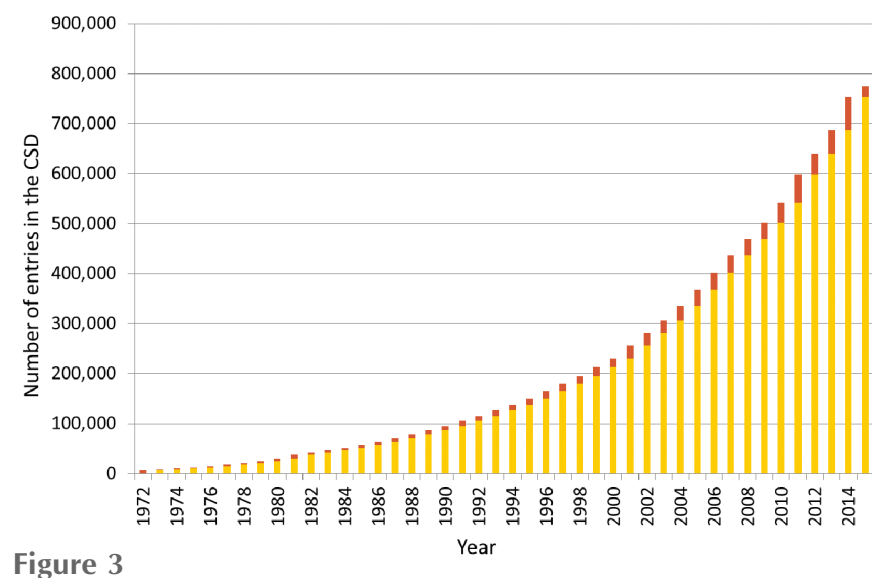

Figure 3

Entries in the CSD 1972-2015 (Ward, 2015). The red color indicates structures added annually. named reactions and these have typically been developed and refined through extensive and lengthy optimization procedures. Many will only work on a narrow range of substrates and they often require very specific reaction conditions or custom designed catalysts. Despite the fact that organic synthesis is a mature science, many reactions cannot be made to provide the desired pure product in a high-yielding manner. However, if a particular transformation produces the target molecule in reasonable quantities, we would consider this to be a successful effort.

Similarly, co-crystallizations between two, or more, different molecular building blocks require that careful attention be paid to solvent, to the relative solubility of the reactants, and to the way in which the solid is isolated. Furthermore, the presence of competing functional groups on a substrate can lead to unwanted 'synthon-crossover' (Aakeröy et al., 2011) which will derail a synthetic strategy in pursuit of a specific supramolecular target. However, if a particular synthon is capable of producing co-crystals having the intended stoichiometry and targeted motif with a reasonably high frequency of occurrence, with a high supramolecular yield (Aakeröy et al., 2001, 2002), then there is no reason why we should not also consider this to be a successful synthetic effort. An approach that has been particularly helpful for circumventing problems with solubility differences between reactants in co-crystal synthesis is to employ solvent-assisted or liquidassisted grinding protocols, approaches which are often grouped together under the umbrella of mechanochemical synthesis. The underlying principles behind mechanochemical synthetic process have often been examined very productively on co-crystal systems (Shan et al., 2002; Braga et al., 2013), and this technology is receiving considerable attention in the context of both organic/inorganic synthesis and well as against the backdrop of green chemistry (James \& Friščić, 2013).

On the other hand, one should not shy away from the fact that a large number of publications truly are 'routine' and merely offer a crystal-structure report, maybe accompanied by measurements of some rather random physical properties, without any design strategy in place or without discussing the new structural data in a larger relevant context. Exploring the 'structural diversity' of very flexible molecules with a large number of potential binding sites without having a supramolecular synthetic target in mind or without presenting experiments in response to a hypothesis is unlikely to produce insight and understanding and typically will only amount to a purely descriptive outcome. Again, much more crystallographic data is absolutely needed in order to move the field forward and allow us to develop more robust and versatile synthetic strategies, but little is gained by pretending that a straightforward report on one or more crystal structures represents a full-blown research article that describes deliberate and directed co-crystal synthesis.

\section{Applications of co-crystals and co-crystal technology}

The field of applied science where co-crystals and co-crystal technology have been of most interest is undoubtedly in the 
pharmaceutical arena. This is probably to be expected as, in general, the most favored medium for an active pharmaceutical ingredient (API) is the crystalline solid state partly because of reasons related to chemical purity, stability and shelf-life. The particular solid form of an API also governs key properties such as solubility, dissolution rate and thermal stability, all of which determine the overall performance of the drug. In addition, the physical properties of the solid also determine the type of delivery methods and formulations that are going to be available for a particular drug (Almarsson \& Zaworotko, 2004). The pharmaceutical industry is often very cautious and slow to react to innovation and new ideas that originate at a fundamental academic level. Consequently, it is therefore quite surprising that concepts and terminologies associated with co-crystals have already had an impact on the way in which many pharma companies consider co-crystal screens as part of their general work flow in the pursuit of more effective formulations (in much the same way that salt screens and polymorph screens are part and parcel of the standard evaluation of the solid form of most drugs). In principle, co-crystals can offer avenues to produce new solid forms of an API with substantially different (and improved) properties (Babu et al., 2012) as well as providing legal opportunities for either circumventing a patent or for extending the lifetime of an existing invention (Almarsson et al., 2012). Numerous US and EP patents have been filed on cocrystals of pharmaceutical relevance both in the US and in Europe since 2000 and these focus typically either on methodology or composition. The flurry of activity surrounding cocrystal technologies in a pharmaceutical context has produced real and tangible momentum and there is little doubt that we can expect to see several existing or new drugs formulated as co-crystals pass through clinical trials and enter the market within a relatively short time span. Although co-crystals of APIs are not going to be a panacea [for example, it seems unlikely that they will, in general, be any less prone to polymorphism than are homomeric molecular solids (Aitipamula et al., 2014)], they will likely offer useful strategies and opportunities that can facilitate the development of new drugs and formulations for the future. Similar progress can be expected in areas related to agrochemicals (George et al., 2013), nutraceuticals (Schultheiss et al., 2010), liquid crystals (Nguyen et al., 2004), charge-transfer (Zhu et al., 2015) and non-linear optical materials (Gryl et al., 2015), to name but a few.

Finally, impact sensitivity and stability of an explosive can often be related to the presence and relative orientation of specific crystallographic parameters. Consequently, it makes sense that productive avenues towards new and improved energetic materials may be found through the use of co-crystal technology (Anderson et al., 2014; Li et al., 2014), whereby an energetic material is combined with either an energetic or a non-energetic compound (Guo et al., 2013) via non-covalent interactions within a crystalline framework (Landenberger $e t$ al., 2013). All in all, a co-crystal version of an energetic material can, in certain circumstances, be more useful because of superior chemical stability and shelf-life even though it may have slightly lower energetic performance. More generally, cocrystallizations may also offer an avenue for transforming liquid high-value chemicals into crystalline materials with low vapor pressure, considerable thermal stability, and moisture resistance that can readily be regenerated through simple solvent extractions (Aakeröy, Welideniya \& Desper, 2015).

\section{Unique advantages of co-crystals}

One of the most important aspects of co-crystals is that they can offer a degree of predictable structural periodicity that is essential to the design and synthesis of materials with truly tunable (and not just changeable) physical properties. The extent to which a series of co-crystals provides a useful framework for realising a particular property will increase as the dimensionality of the dominating supramolecular motif increases. If the primary non-covalent interactions responsible for the co-crystal synthesis essentially leads to discrete supramolecular entities (dimers, trimers etc), then there is little advantage for heteromeric co-crystals over homomeric molecular solids. A discrete supermolecule will possess as many degrees of freedom as a single molecule does, and without structural consistency and periodicity it will be very difficult to a priori relate molecular structure to bulk properties. However, if the co-crystal is composed of robust infinite chains or layers the number of possible structural variations in a series of co-crystals becomes much more limited. Consequently, if one reactant $(A)$ is kept constant and the other reactants belong to a family of molecules $\left(B_{1}, B_{2}, B_{3}\right.$ etc. $)$ that are likely to form consistent and reproducible synthons then the chance of correlating molecular structure (or even properties of the bulk material of the individual homomeric solids) with one or more physical properties of the co-crystal is improved dramatically. Robust synthons can be used to build a reliable framework which can act as a de facto, crystalline, infinite host molecule that, in turn, can accommodate different members of a synthon-consistent family with very little structural change, Fig. 4.

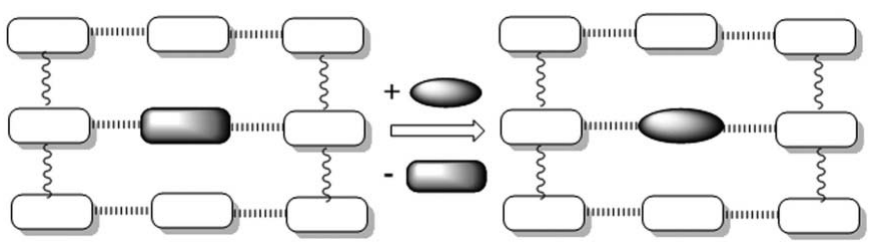

Figure 4

Suitably complementary synthons can produce structurally consistent architectures where one of the co-formers can be replaced with little or no detrimental structural consequences. As the overall structures remain the same, the replacement of one building block with another can offer genuine tunability of physical properties and by taking advantage of this unique modularity within the context of structural consistency a specific property or macroscopic response may be precisely 'dialed-in' akin to what can be achieved with alloys in metallurgy and by doping in semiconductors. 


\section{Codicil}

Practical co-crystal synthesis utilizes a bottom-up approach towards the assembly and construction of large architectures with well defined chemical compositions, topologies and dimensions. The building blocks themselves are primarily going to be discrete chemical entities such as neutral organic molecules. The deliberate and directed assembly of these entities into larger structures is achieved with the aid of a wide variety of non-covalent interactions including (but not limited to) $\pi \cdots \pi$ interactions and hydrogen and halogen bonds. As such, this subject cuts across the traditional divisions of organic, inorganic and physical chemistry and this produces an eclectic blend of ideas, techniques and strategies.

What we are learning from fundamental studies on the synthesis and characterization of co-crystals is already positively affecting the design of new functional solids, but equally important is the task of seeking a better understanding of how fundamental laws of physics manifest themselves in ordered crystalline materials. Progress in this area will take place at the confluence of organic, inorganic, physical and theoretical chemistry and requires a unique interface of experimental and theoretical tools and data provided by academia as well as by industry. Co-crystals represent new classes of compounds where bulk physical properties may be amenable to finetuning by making modular and controllable alterations to the crystalline lattice that 'houses' an active molecular species. The links between crystal structure, morphology and solidstate properties may also offer opportunities for improving processing, performance and shelf-life of a wide range of specialty chemicals. Consequently, an ability to control and change the crystalline environment of a material without altering molecular properties would be of enormous significance to manufacturers and consumers alike. With this in mind, it would seem that the answer to the question posed at the beginning of this manuscript is indeed 'Yes'.

\section{Acknowledgements}

First, I would like to express sincere gratitude to my students, past and present, for their enduring efforts and enthusiasm even when faced with some rather ill-conceived project ideas. Second, thank you to the wonderful mentors, collaborators and friends that I have met and worked with over the years. The pursuit of new science is important, but it would be a much less enjoyable quest without the people that appear along the way.

\section{References}

Aakeröy, C. B., Beatty, A. M. \& Helfrich, B. A. (2001). Angew. Chem. Int. Ed. 40, 3240-3242.
Aakeröy, C. B., Beatty, A. M. \& Helfrich, B. A. (2002). J. Am. Chem. Soc. 124, 14425-14432.

Aakeröy, C. B., Chopade, P. D. \& Desper, J. (2011). Cryst. Growth Des. 11, 5333-5336.

Aakeröy, C. B. \& Salmon, D. J. (2005). CrystEngComm, 7, 439448.

Aakeröy, C. B., Welideniya, D. W. \& Desper, J. (2015). Personal communication.

Aakeröy, C. B., Wijethunga, T. K., Benton, J. \& Desper, J. (2015). Chem. Commun. 51, 2425-2428.

Aitipamula, S., Chow, P. S. \& Tan, R. B. H. (2014). CrystEngComm, 16, 3451-3465.

Allen, F. H. (2002). Acta Cryst. B58, 380-388.

Almarsson, Ö., Peterson, M. L. \& Zaworotko, M. J. (2012). Pharm. Pat. Anal. 1, 313-327.

Almarsson, Ö. \& Zaworotko, M. J. (2004). Chem. Commun. 17, 1889 1896.

Anderson, S. R., Am Ende, D. J., Salan, J. S. \& Samuels, P. (2014). Propellants Explosives Pyrotech. 39, 637-640.

Babu, N. J., Sanphui, P. \& Nangia, A. (2012). Chem. Asian J. 7, 2274 2285.

Bond, A. D. (2007). CrystEngComm, 9, 833-834.

Bosch, B. (2014). Cryst. Growth Des. 14, 126-130.

Braga, D., Maini, L. \& Grepioni, F. (2013). Chem. Soc. Rev. 42, 76387648.

Bruno, I. J. \& Groom, C. R. (2014). J. Comput. Aided Mol. Des. 28, 1015-1022.

Childs, S. L. \& Zaworotko, M. J. (2009). Cryst. Growth Des. 9, 42084211.

Desiraju, G. R. (1989). Crystal Engineering: the Design of Organic Solids. Amsterdam, New York: Elsevier.

Desiraju, G. R. (1995). Angew. Chem. Int. Ed. Engl. 34, 2311-2327.

Desiraju, G. R. (2003). CrystEngComm, 5, 466-467.

Dunitz, J. D. (2003). CrystEngComm, 5, 506.

Etter, M. C. (1990). J. Phys. Chem. 95, 4601-4610.

George, N., Forrest, J. O., Burton, R. C. \& Aakeröy, C. B. (2013). US Patent US2013/0203792A1, 8 August 2013.

Gryl, M., Cenedese, S. \& Stadnicka, K. (2015). J. Phys. Chem. C, 119, $590-598$.

Guo, C., Zhang, H., Wang, X., Liu, X. \& Sun, J. (2013). J. Mater. Sci. 48, 1351-1357.

Jacobellis versus Ohio (1964). 378 U.S. 184.

James, S. L. \& Friščić, T. (2013). Chem. Soc. Rev. 42, 7494-7496.

Landenberger, K. B., Bolton, O. \& Matzger, A. J. (2013). Angew. Chem. Int. Ed. 52, 6468-6471.

Li, H. R., Shu, Y. J., Song, C., Chen, L., Xu, R. J. \& Ju, X. H. (2014). Chin. Chem. Lett. 25, 783-786.

Nguyen, H. L., Horton, P. N., Hursthouse, M. B., Legon, A. C. \& Bruce, D. W. (2004). J. Am. Chem. Soc. 126, 16-17.

Schultheiss, N., Bethune, S. \& Henck, J.-O. (2010). CrystEngComm, 12, 2436-2442.

Shan, N., Toda, F. \& Jones, W. (2002). Chem. Commun. pp. 23722373.

Stahly, G. P. (2009). Cryst. Growth Des. 9, 4212-4229.

Tothadi, S. \& Desiraju, G. R. (2013). Chem. Commun. 49, 77917793.

Ward, S. (2015). Personal communication.

Wuest, J. D. (2001). J. Am. Chem. Soc. 113, 4696-4698.

Zhu, W., Zheng, R., Fu, X., Fu, H., Shi, Q., Zhen, Y., Dong, H. \& Hu, W. (2015). Angew. Chem. Int. Ed. 54, 6785-6789. 